\title{
Clinical and pathological characteristics of IgG4-related interstitial lung disease
}

\author{
XIAOTING LV ${ }^{1,2}$, FENG GAO ${ }^{3}$, QICAI LIU ${ }^{4}$, SHENG ZHANG $^{3}$, ZHIHUA HUANG $^{1,2}$, \\ YONGPING ZHU ${ }^{5}$, HAIYANG ZONG ${ }^{6}$, QUWEN $\mathrm{LI}^{7}$ and SANYAN LI ${ }^{3}$ \\ ${ }^{1}$ Respiratory Disease Research Laboratory; Departments of ${ }^{2}$ Respiration, ${ }^{3}$ Pathology and ${ }^{4}$ Laboratory Medicine, \\ The First Affiliated Hospital, Fujian Medical University; ${ }^{5}$ Department of Cardiovascular Surgery, The Union Hospital, \\ Fujian Medical University, Fuzhou, Fujian 350005; ${ }^{6}$ Department of Orthopaedic Surgery, Changzheng Hospital, \\ The Second Military Medical University, Shanghai 200003; ${ }^{7}$ Priority Laboratory for Zoonoses Research, \\ Fujian Centre for Disease and Prevention, Fuzhou, Fujian 350001, P.R. China
}

Received May 5, 2017; Accepted October 6, 2017

DOI: $10.3892 /$ etm.2017.5554

\begin{abstract}
IgG4-related interstitial lung disease (IgG4-RILD), which is characterized by increased IgG4 levels, IgG4 ${ }^{+}$plasma cell infiltration and irregular whorled fibrosis, is a recently described lung disorder that belongs to the group of systemic fibroinflammatory IgG4-related diseases (IgG4-RD). The aim of the present study was to improve the current knowledge regarding the specific clinical and histopathological characteristics of IgG4-RILD and to investigate its underlying immune mechanism in vivo. A total of 7 patients newly diagnosed with IgG4-RILD were enrolled in the present study (4 men and 3 women; mean age, 57 years; range, 29-71 years). Patients' clinical history was collected and serological indicators, including C-reactive protein (CRP), erythrocyte sedimentation rate (ESR), antinuclear antibodies (ANAs) and tumor markers were measured. Serum immunoglobulin G (IgG), IgE and IgG4 levels were also evaluated. In addition, computed tomographic (CT) images and pathological examinations were used to determine the characteristics of lung lesions in all patients. The majority of patients presented with symptoms of fever, cough and dyspnea, while allergic symptoms were also encountered. The laboratory examination results revealed different degrees of increased CRP, ESR, tumor markers, ANA, serum IgE and IgG4. The CT images revealed diffuse ground glass opacities, bronchiectasis and thickened bronchovascular bundles. Histologically, the lung lesions were characterized by dense IgG4 ${ }^{+}$lymphoplasmacytic infiltrates intermixed with extensive fibrous tissue hyperplasia and an irregularly storiform pattern of fibrosis. The mean number of $\operatorname{IgG} 4^{+}$plasma cells
\end{abstract}

Correspondence to: Dr Qicai Liu, Department of Laboratory Medicine, The First Affiliated Hospital, Fujian Medical University, 20 Chazhong Road, Taijiang, Fuzhou, Fujian 350005, P.R. China E-mail: 1qc673673673@126.com

Key words: interstitial lung disease, autoimmune diseases, immunoglobulin G4, fibrosis, immunoglobulin G4-related diseases was $>10$ cells/high power field. The ratio of $\mathrm{IgG} / \mathrm{IgG} 4^{+}$plasma cells was $>50 \%$ in inflamed lesions and the number of parenchymal cells was markedly reduced. Obliterative phlebitis or obliterative arteritis was observed in all patients. In conclusion, the clinicopathological similarities between IgG4-RILD and other IgG4-RD suggest that IgG4-related immunopathological processes may be associated with the pathogenesis of pulmonary lesions. Future studies based on the findings herein may elucidate the specific pathological process underlying the development of this fibroinflammatory disorder.

\section{Introduction}

IgG4-related disease (IgG4-RD) is a newly characterized group of systemic fibroinflammatory diseases with elevated serum IgG4 levels (1-3). It was first described in Japan as type 1 autoimmune pancreatitis (AIP) presenting with obstructive jaundice due to a tumefactive lesion of the pancreas $(4,5)$; however, it was subsequently identified in almost all organs and referred to by a number of names, including Mikulicz's disease, sclerosing cholangitis and IgG4-related lung disease (6-9). These IgG4-RD share similar pathological characteristics, including distinctive fibrosis with an irregular whorled pattern (storiform fibrosis), obliterative phlebitis/arteritis and prominent lymphoplasmacytic (mostly $\mathrm{IgG}^{+}$plasma cells) tissue infiltration (8). However, the pathogenesis of IgG4-RD is poorly understood.

Respiratory involvement of IgG4-RD has previously been described, and manifestations of lung involvement, including IgG4-related interstitial lung disease (IgG4-RILD), have been attracting increasing attention based on clinical and radiographic evidence $(7,10,11)$. Furthermore, it has been demonstrated that interstitial pneumonia (IP) in IgG4-related lung disease may coexist with inflammatory pseudotumor (IPT; plasma cell granuloma) lesions at the boundaries of nodules (7). Accordingly, IgG4-RILD is often overlooked or misdiagnosed as non-specific IP (NSIP), another ILD or lung cancer $(6,10,12,13)$. Although patients with IgG4-RD typically respond well to corticosteroid therapy (10), incorrect diagnoses may lead to unnecessary surgical intervention and delayed 
administration of effective corticosteroid treatment in the early stages of the disease, which may prove life threatening for the patient.

The aim of the present study was to determine the specific clinical and histopathological characteristics of IgG4-RILD and to investigate its underlying immune mechanism. Relevant cases of IgG4-RILD were assessed in order to improve diagnostic and treatment methods for this disease.

\section{Patients and methods}

Patient characteristics. A retrospective study was undertaken to investigate patients hospitalized with IgG4-RILD. A total of 7 cases were selected at the First Affiliated Hospital between August 2012 and December 2016, as defined in the comprehensive diagnostic criteria for IgG4-RD guidelines (14). Furthermore, interstitial lung disorder was identified by chest CT scanning or pathological examination. The patients were aged 29-71 years and included 4 men and 3 women. Patients with malignant tumors, multicentric Castleman's disease and Sjogren's disease, as defined in the IgG4-RD guidelines, were excluded (14-16). Clinical records were available for all patients and were reviewed for clinical symptoms on presentation and laboratory data. Patients with pulmonary bullae served as control subjects. Patients with newly diagnosed pulmonary bullae $(n=20)$ were enrolled (17 males and 3 females; mean age 56 years; range, 45-71 years) at the First Affiliated Hospital between April 2015 and December 2016, diagnosis was confirmed by lung CT images. Patients with malignant tumors, bronchiectasis and pulmonary cystic fibrosis were excluded. The present study was approved by the Medical Ethics Committee of the First Affiliated Hospital of Fujian Medical University (Fujian, China).

Laboratory data. Serum IgG4 levels were determined with the use of a commercially available immunoturbidimetry assay kit (BNII Nephelometer; Siemens Healthineers, Erlangen, Germany) according to the manufacturer's instructions. The following laboratory data were also analyzed in all patients using the respective kits according to the manufacturer's instructions: C-reactive protein (CRP), erythrocyte sedimentation rate (ESR), IgG, antinuclear antibodies (ANAs) (Euroimmun AG, Lübeck, Germany) and tumor markers [carcinoembryonic antigen (CEA; cat. no. 218910), cancer antigen 125 (cat. no. 187489; CA125), and cancer antigen 199 (CA199; cat. no. 231612) (all from Roche Applied Science, Rotkreuz, Switzerland)]. CRP, ESR and IgG were measured using immune turbidimetry (BNII Nephelometer; Siemens Healthineers). CEA, CA125, CA199 and IgG were detected with electrochemiluminescence (cobas e 601; Roche Applied Science).

Hematoxylin and eosin staining. Of the 7 patients, 1 (case 1) underwent surgical resection of the pulmonary lesions due to suspicion of lung cancer and 6 patients (cases 2-7) underwent CT-guided fine-needle aspiration biopsy of the lung lesions. Lung tissue samples from each surgically resected or biopsied specimen were fixed in $4 \%$ formalin overnight at $25^{\circ} \mathrm{C}$, embedded in paraffin, sectioned at $5 \mathrm{~mm}$ thickness and stained with hematoxylin and eosin for pathology. Paraffin embedding was carried out at $60^{\circ} \mathrm{C}$ for $30 \mathrm{~min}$ in four $100 \%$ paraffin steps. At $40^{\circ} \mathrm{C}$, lung sections were deparaffinized in xylene twice for 5 min each time and rehydrated in a series of graded ethanol (70, 80, 96 and $100 \%$ ). At $25^{\circ} \mathrm{C}$, sections were subsequently stained with hematoxylin for $2 \mathrm{~min}$, rinsed with distilled water, rinsed with $0.1 \%$ hydrochloric acid in $50 \%$ ethanol for $15 \mathrm{~min}$, stained with eosin for $1 \mathrm{~min}$ and rinsed again using distilled water. Slides were dehydrated with 95 and $100 \%$ ethanol and mounted with coverslips. Sections were then examined under a light microscope (DWLB2; Leica Microsystems GmbH, Wetzlar, Germany; magnification, x400).

Immunohistochemical studies. Paraffin-embedded sections $(5 \mathrm{~mm})$ from patients with IgG4-RILD and pulmonary bullae were routinely dewaxed and rehydrated and then incubated with $3 \% \mathrm{H}_{2} \mathrm{O}_{2}$ to block endogenous catalase for $10 \mathrm{~min}$. Antigen retrieval was performed by heating the slides in citrate buffer at $100^{\circ} \mathrm{C}$ for $20 \mathrm{~min}$. Samples were subsequently incubated with the following primary antibodies at $37^{\circ} \mathrm{C}$ for $60 \mathrm{~min}$ : Anti-cluster of differentiation (CD)3 (1:200; cat. no. A1753), anti-CD20 (1:500; cat. no. A1793), anti-CD38 (1:2,000; cat. no. A1680), anti-CD68 (1:200; cat. no. A6554) (all from ABclonal Biotech Co., Ltd., Woburn, MA, USA) and anti- $\alpha-1$-antitrypsin ( $\alpha$-AT; 1:100; cat. no. M00720; Wuhan Boster Biological Technology, Ltd., Wuhan, China). Horseradish peroxidase-conjugated secondary antibody (1:2,000; cat. no. AS014; ABclonal Biotech Co., Ltd.) was used to mark the primary antibody. Following washing three times with phosphate-buffered saline (PBS), the sections were incubated with secondary antibody for $30 \mathrm{~min}$ at $25^{\circ} \mathrm{C}$, followed by the addition of the color reagent, 3,3'-diaminobenzidine $\mathrm{DAB}$ for $10 \mathrm{~min}$ at $25^{\circ} \mathrm{C}$ and counterstaining with hematoxylin for $1 \mathrm{~min}$ at $25^{\circ} \mathrm{C}$. Sections from patient and control samples were examined under a light microscope (DWLB2; Leica Microsystems GmbH; magnification, x400) and images were captured from five random fields. The mean number of stained $\mathrm{IgG} 4^{+}$plasma cells in each section was counted and the ratio of stained $\mathrm{IgG} 4^{+}$cells to total $\mathrm{IgG}^{+}$cells was calculated. The histological findings were assessed by two pathologists.

Immunofluorescent staining. Lung tissue samples from patients with IgG4-RILD and pulmonary bullae were fixed in $4 \%$ formalin overnight at $25^{\circ} \mathrm{C}$, embedded in paraffin and sectioned at $5 \mathrm{~mm}$ thickness. Sections adjacent to those used in immunohistochemical analysis were blocked in 10\% normal animal serum (cat. no. AR0009; Wuhan Boster Biological Technology, Ltd.) for $20 \mathrm{~min}$ at room temperature. They were subsequently incubated with the following primary antibodies overnight at $4^{\circ} \mathrm{C}$ : Collagen IV (1:2,000; cat. no. A7657; ABclonal Biotech Co., Ltd.) and IgG4 (1:1,000; cat. no. ab109493; Abcam, Cambridge, UK). Following washing 3 times with PBS containing $0.1 \%(\mathrm{v} / \mathrm{v})$ Tween-20, sections were incubated with a Cy3 goat anti-rabbit IgG antibody (red, 1:400; cat. no. AS008; ABclonal Biotech Co., Ltd.) or a FITC goat anti-rabbit IgG antibody (green, 1:100; cat. no. AS011; ABclonal Biotech Co., Ltd.) for $1 \mathrm{~h}$ at $25^{\circ} \mathrm{C}$. Sections were then counterstained with 4',6-diamidino-2-phenylindole (DAPI; 1:100; cat. no. AR1177; nuclear staining; Wuhan Boster Biological 

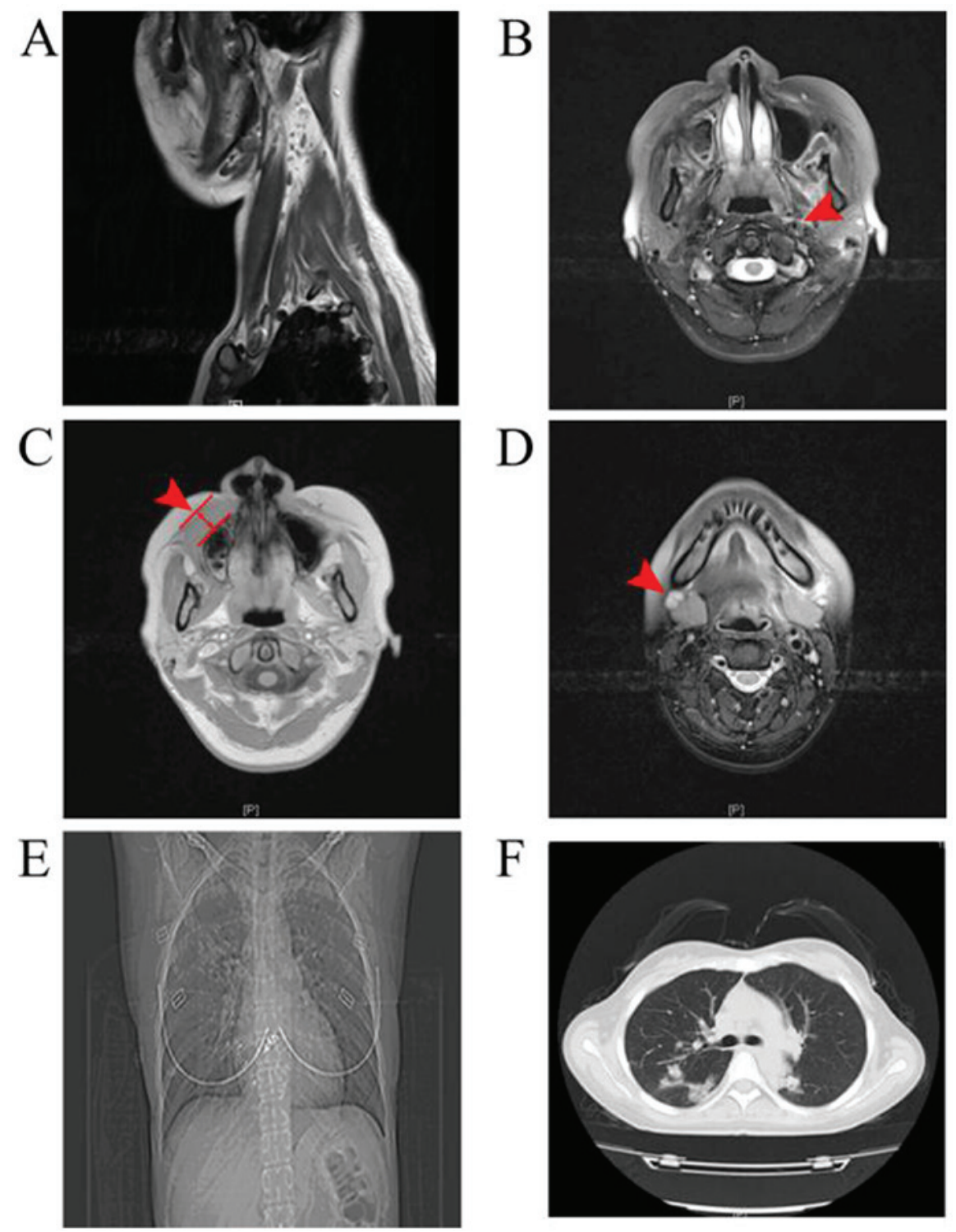

Figure 1. Image scans of systemic fibroinflammatory lesions in case 1 with immunoglobulin G4-related disease. (A) Head and neck were scanned with MRI plain scan by anteroposterior axes position. (B-D) Images were scanned with MRI plain scan in the horizontal position. (B) Inflammatory pseudotumor of the parotid gland was observed on the left side (red arrow). (C) An orbital inflammatory pseudotumor (red arrow) was also identified. (D) An enlarged mandibular lymph node was observed (red arrow). (A-D) Image scans showed chronic sclerosing sialadenitis in case 1. (E) Lung CT images were scanned in the coronal position. (F) Diffuse ground glass opacity, marked bronchiectasis, thickened bronchovascular bundles and multiple small nodules were observed in both lungs with CT plain scan by horizontal position. MRI, magnetic resonance imaging; CT, computed tomography.

Technology, Ltd.) for $20 \mathrm{~min}$ at $25^{\circ} \mathrm{C}$. Patient and control samples were examined under a light microscope (DWLB2; Leica Microsystems GmbH; magnification, x400).

Elastica-van Gieson staining. Lung tissue samples from patients with IgG4-RILD and control subjects were fixed in $4 \%$ formalin overnight at $25^{\circ} \mathrm{C}$, embedded in paraffin and sectioned at $5 \mathrm{~mm}$ thickness. Samples, adjacent to those used in immunohistochemical analysis, were then blocked with acidified potassium permanganate at room temperature for $2 \mathrm{~min}$, followed by washing in $1 \%$ oxalic acid for $1 \mathrm{~min}$. At room temperature, sections were stained in Weigert's resorcin-fuchsin for $45 \mathrm{~min}$. Then, samples were flushed with water for $10 \mathrm{~min}$ at $25^{\circ} \mathrm{C}$, differentiated in acid alcohol and stained with Van Gieson for $5 \mathrm{~min}$. Sections from the patient and control samples were then examined under a light microscope (DWLB2; Leica Microsystems GmbH; magnification, $\mathrm{x} 400)$.

\section{Results}

Clinical history. Radiological abnormalities observed during CT scanning were similar for all patients and IgG4-RILD was observed on pathological examination of lung specimens (cases 1-7). Extrapulmonary IgG4-RD was identified by means of biopsy in 5 patients: Acute pyelonephritis (case 1), chronic sclerosing sialadenitis (cases 1 and 5; Fig. 1A-D), IgG4-related skin lesions (cases 1 and 3), and AIP (cases 6 and 7). Allergic symptoms, including chronic eczema and allergic purpura, were also observed in cases 1 and 3 (Table I). Elevated CRP levels were detected in all cases to varying degrees, with the exception of case 6 (range, 3.4-122 mg/l; 


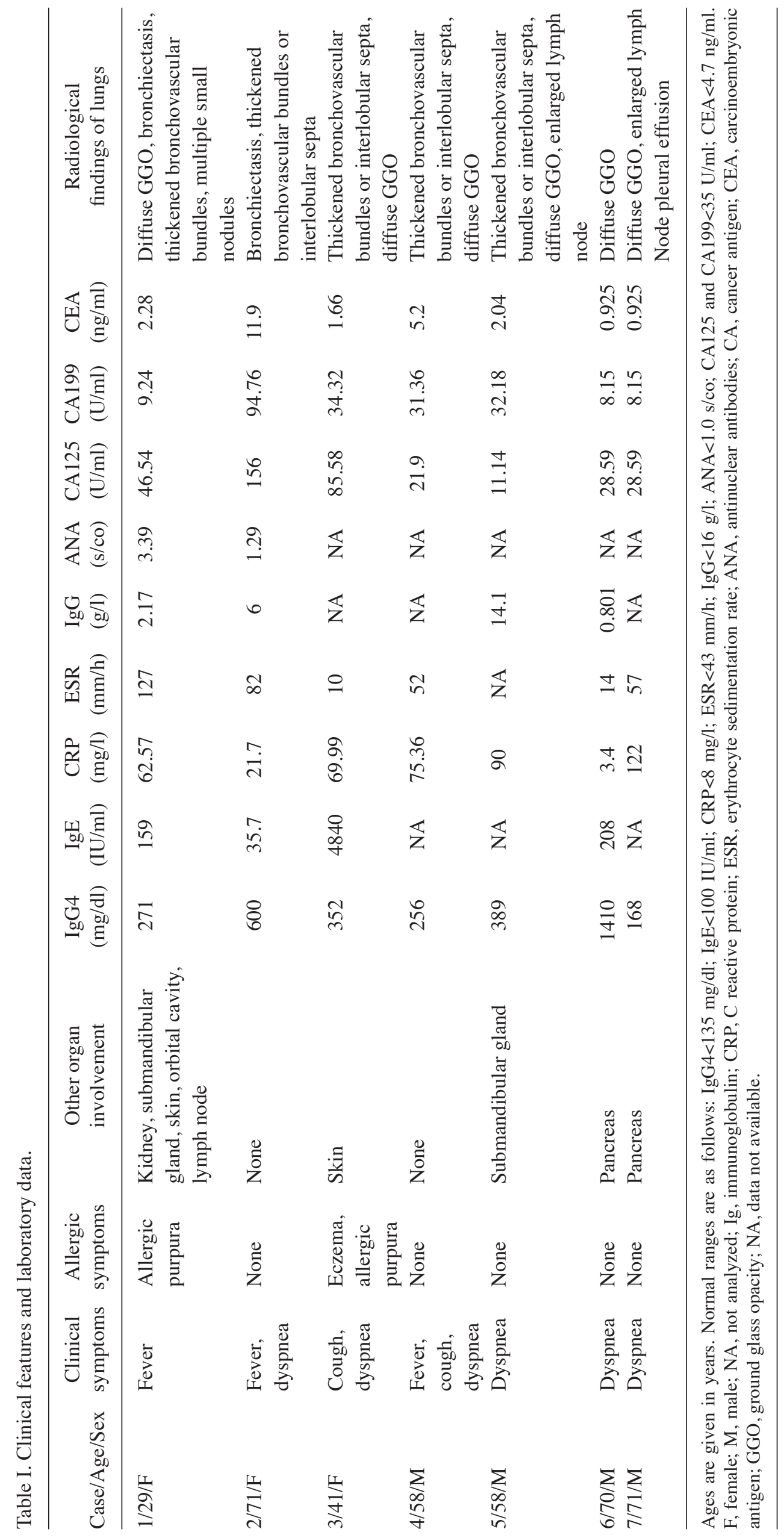



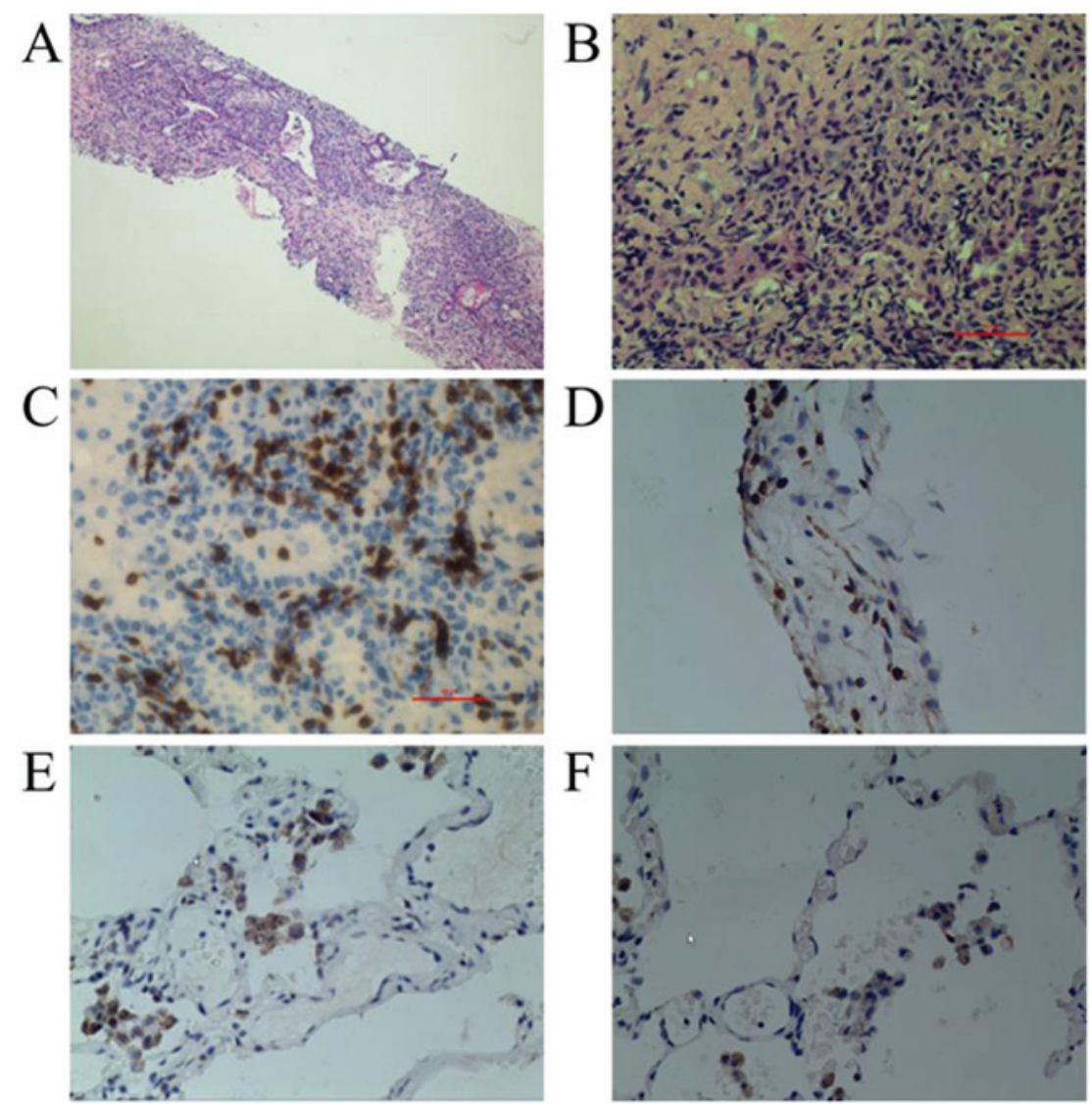

Figure 2. Histological characteristics of IgG4-related lung disease in case 1. (A) Solid nodular lesions stained with H\&E had a mixed inflammatory infiltrate and fibrosis. Magnification, x100. (B) Inflammatory cells stained with H\&E mainly consisted of lymphocytes and plasma cells. Some eosinophils were also observed and the number of alveolar epithelial cells was markedly reduced. Magnification, x400. (C) IgG4 immunohistochemical staining revealed numerous immunoreactive plasma cells. Magnification, x400. (D) Immunohistochemical analysis of the expressions of CD3 in serial sections of IgG4-related lung disease. Magnification, x400. (E) Immunohistochemical analysis of the expression of CD20 in serial sections of IgG4-related lung disease. Magnification, x400. (F) Immunohistochemical analysis of the expressions of CD38 in serial sections of IgG4-related lung disease. Magnification, x400. H\&E, hematoxylin and eosin; IgG4, immunoglobulin G4; CD, cluster of differentiation.

normal, $<8 \mathrm{mg} / \mathrm{l})$. Elevated ESR was detected in cases 1, 2, 4 and 7 (range, $10-127 \mathrm{~mm} / \mathrm{h}$; normal, $<43 \mathrm{~mm} / \mathrm{h}$ ). Serum $\mathrm{IgG}$ levels (range, $0.801-14.1 \mathrm{~g} / 1$; normal, $<16 \mathrm{~g} / \mathrm{l}$ ) were available for cases 1, 2, 5 and 6, while ANA levels (range, 1.29-3.39 s/co; normal, $<1.0 \mathrm{~s} / \mathrm{co}$ ) were only measured in cases 1 and 2 . As a retrospective study, the data in the present study were not collected for all the patients. The numerical value of serum CA125 (range, 11.14-156U/ml; normal, $<35 \mathrm{U} / \mathrm{ml}$ ) was increased in cases 1-3. High levels of CA199 (range, 8.15-94.76 U/ml; normal, $<35 \mathrm{U} / \mathrm{ml}$ ) and CEA (range, $0.925-11.9 \mathrm{ng} / \mathrm{ml}$; normal, $<4.7 \mathrm{ng} / \mathrm{ml}$ ) were observed in case 2 . Case 4 also exhibited high expression of CEA. Serum IgG4 in these patients was high (range, $168-1410 \mathrm{mg} / \mathrm{dl}$; normal, $<135 \mathrm{mg} / \mathrm{dl}$ ) and the level of IgE was above normal (range, 35.7-4840 IU/ml; normal, $<100 \mathrm{IU} / \mathrm{ml}$ ) (Table I).

Imaging analysis. Patients were categorized into four major subtypes based on the predominant radiological abnormality observed: Solid nodular type, exhibiting a solitary nodular mass lesion; round-shaped ground-glass opacity (GGO) type, characterized by multiple round-shaped GGO; alveolar interstitial type, displaying honeycombing, bronchiectasis and diffuse GGO; and bronchovascular type, exhibiting thickening of bronchovascular bundles and interlobular septa (11). The
CT images revealed alveolar interstitial and bronchovascular types, which were evaluated for the presence and distribution of GGO (cases 1 and 3-7), multiple small nodules (case 1; Fig. 1E and F), thickening of bronchovascular bundles or interlobular septa (cases 1-5), bronchiectasia (cases 1 and 2), pleural effusion (case 7) and enlarged lymph nodes (cases 5 and 7). No honeycombing, cysts or round-shaped GGO were observed in these cases.

Histological findings. In case 1, lung specimens from solid nodular pulmonary lesions exhibited diffuse interstitial thickening with extensive plasma cell infiltration compared with the control group. Solid nodular lesions had a mixed inflammatory infiltrate and fibrosis, and the number of alveolar epithelial cells was markedly reduced (Fig. 2A and B). Following immunostaining of the polyclonal B cells and plasma cells, $\mathrm{IgG}^{+}$ plasma cells were observed to be diffusely distributed. IgG4 ${ }^{+}$ plasma cells were abundant in inflamed areas, including the alveolar interstitium, interlobular septa, and peribronchial or peribronchiolar interstitium (Fig. 2C). Other inflammatory cells, including CD3, CD20, CD38 and CD68 positive cells, were also observed in inflamed areas (Figs. 2D-F and 3A). CD3-positive cells indicated T lymphocytes, while CD20 and CD38 positive cells indicated plasma cells, and other B 


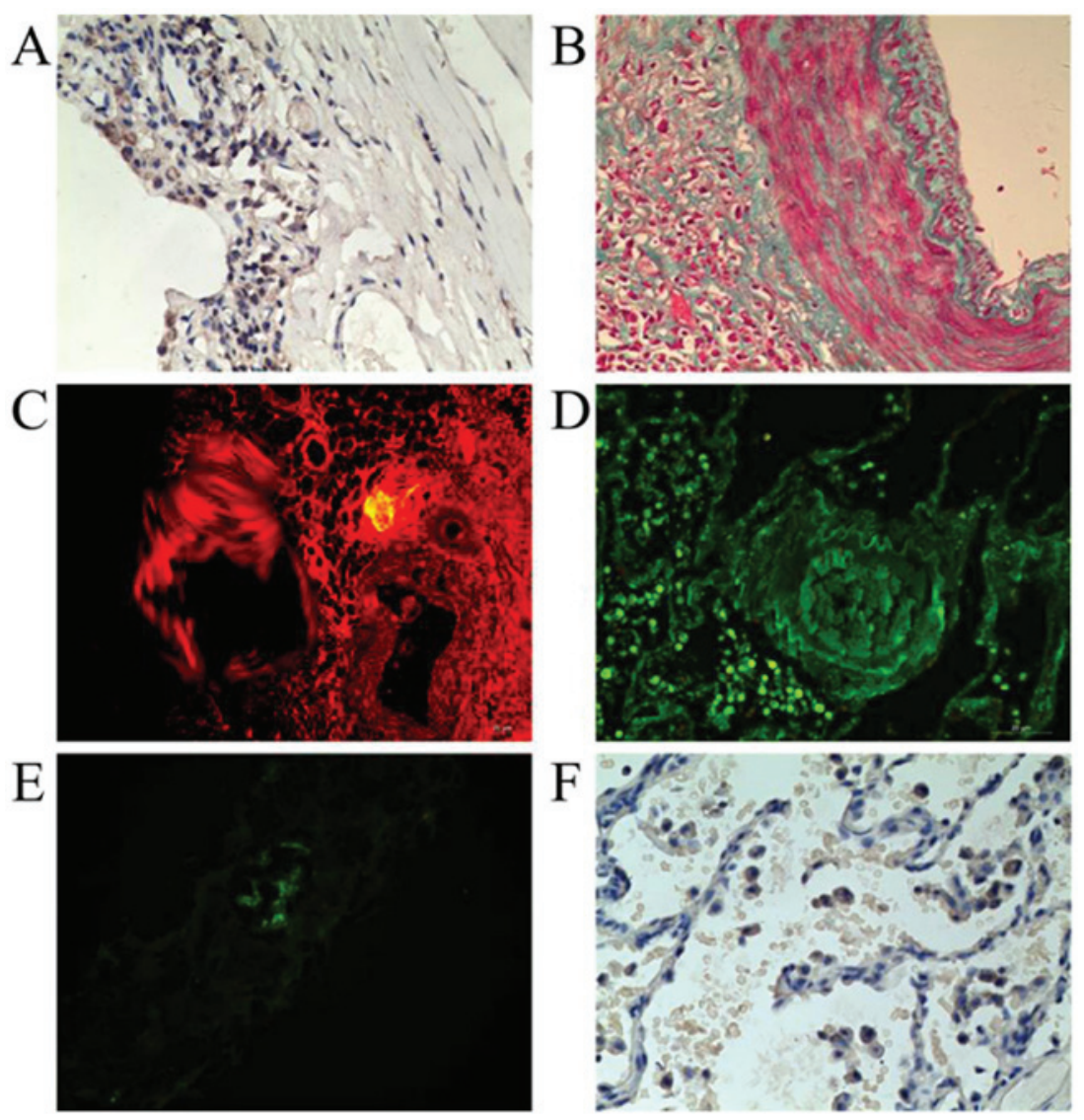

Figure 3. Immunohistochemical analysis of the expression of (A) CD68 in serial sections of IgG4-related lung disease. Magnification, x400. (B) Elastica-van Gieson staining showed collagen fiber diffuses hyperplasia on the vessel wall, followed with collagen aggradation surrounding the vessels. Magnification, $\mathrm{x} 200$. (C) Storiform fibrosis was observed using immunofluorescence staining. Magnification, x400. (D) Immunofluorescence revealed obliterative phlebitis surrounded by lymphoplasmacytic infiltrates at the periphery of the field. Magnification, x400. (E) IgG4 antibody fluorescence of lung tissue surrounding the blood vessels. Magnification, x200. (F) Immunohistochemical analysis of $\alpha-1-\mathrm{AT}$ in IgG4-related lung disease. All samples are from case 1. $\alpha-1-\mathrm{aAT}$, $\alpha$-1-antitrypsin.

lymphocytes and CD68-positive cells indicated macrophages. Furthermore, fibrous tissue hyperplasia and an irregular storiform pattern of fibrosis were observed (Fig. 3B and C). The mean number of $\mathrm{IgG}^{+}$plasma cells was $>10$ cells/HPF in all cases, and the ratio of $\mathrm{IgG} 4 / \mathrm{IgG}^{+}$plasma cells was $>50 \%$ in inflamed lung lesions (Fig. 2C). Furthermore, $\mathrm{IgG}^{+}$plasma cells were observed surrounding the periphery of obliterative phlebitis by immunofluorescent staining (Figs. 3D and E). A pathological diagnosis of IgG4-RILD was confirmed from the obtained lung specimens in all cases. Eosinophils were easily identified (Fig. 2B) and no atypical cells suggestive of a neoplastic process were identified in any of the lesions. The level of $\alpha-1$-AT expression was increased in patients with IgG4-RILD compared with in patients with pulmonary bullae, as shown in Figs. 3F and 4A.

Other pathological findings. Mixed inflammatory infiltrate and fibrosis were observed in other organs (parotid gland and orbit) in case 1; the infiltrate mainly consisted of lymphocytes, plasma cells and some eosinophils, and the number of parenchymal cells was markedly reduced (Fig. 4B and C). In all other cases, lung tissue histological findings were similar to those in case 1 (Fig. 4D-H). Bronchial dilatation was associated with IP and invasive pulmonary Aspergillus infections in case 3 , whereas histochemical staining in the remaining cases revealed no obvious presence of pathogens (Fig. 4I).

\section{Discussion}

IgG4-RILD is considered to be a major subtype of IgG4-related lung disease (7). The number of cases reported worldwide has been increasing gradually with increased understanding and research $(7,10,11)$. However, to the best of our knowledge, there are currently no systematic studies of this disease. The present study was designed to better define the clinical and pathological characteristics of IgG4-RILD and to assess $\mathrm{IgG}^{+}$plasma cells in IgG4-RD and the possible immune mechanisms associated with their presence. The common presenting respiratory symptoms observed among cases were similar to those in previous studies $(7,10,11)$. However, allergic symptoms were relatively common among the cases presented in this study and patients exhibited increased serum indicators, including inflammatory and immunological indices. CT images revealed diffuse interstitial lesions corresponding to histopathological changes of dense IgG4 $4^{+}$lymphoplasmacytic infiltration and an irregularly storiform pattern of fibrosis.

As fibrosing lung diseases encompass a large number of disorders with diverse biological behaviors (17), the intra-pulmonary or extra-pulmonary manifestations in the 


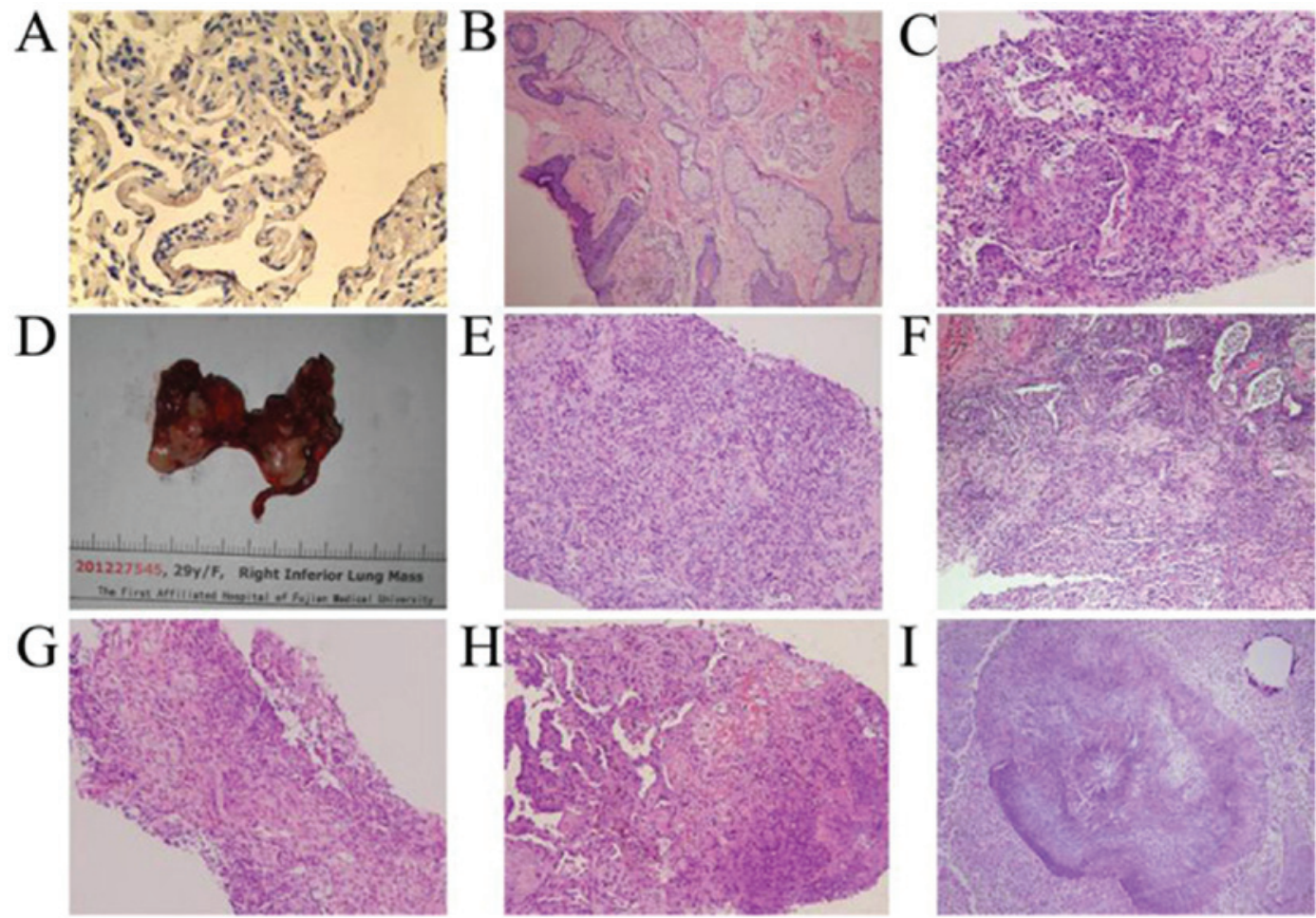

Figure 4. Immunohistochemical analysis of $\alpha$-1-AT in (A) pulmonary bullae and other pathological findings of IgG4-related lung disease. (A) Immunohistochemical analysis of $\alpha-1-A T$ in pulmonary bullae. (B) H\&E staining revealed interstitial inflammation around the orbit with a small number of mature infiltrating lymphocytes. (C) Abundant numbers of inflammatory cells, comprising lymphocytes, plasma cells and eosinophils, in the parotid gland in case 1. Magnification, x200. (D) Gross surgical specimen (lung) from case 1. (E) H\&E staining of histological samples from case 1. Magnification, x100. (F) H\&E staining revealed a large number of lymphocytes, plasma cells and monocytes were identified in the interstitial fibrous tissue of case 3, and the alveolar cavity was filled with myofibroblasts accompanied by partial squamous metaplasia of the cilia in the tracheal epithelium. Magnification, x200. (G) H\&E staining revealed dense lymphoplasmacytic infiltrates intermixed with fibrosis and glandular epithelial atrophy were identified in lung samples in case 3 . Magnification, x200. (H) H\&E staining revealed fibrous tissue hyperplasia in the alveolar septum was observed along with infiltration by lymphocytes, plasma cells and neutrophils in case 5. Magnification, x200. (I) H\&E staining revealed that bronchial dilatation was associated with interstitial pneumonia and fungal infection (Aspergillus) was present in case 3. Magnification, x200.

present study were not typical. The predominant symptoms, including fever, cough and dyspnea, were non-specific compared with idiopathic IPs (IIPs). Similarly, the levels of tumor markers were increased to some extent similar to IIPs. Tumor markers, including CEA, have been reported to be localized to the metaplastic epithelium lining honeycombed bronchioles and have been recognized as biomarkers in IIPs $(18,19)$. The tumor markers in IgG4-RILD may therefore be specific and correlated with the severity of disease. Furthermore, the findings of the present study demonstrate that IP may occur in isolation as a primary symptom or in association with lesions in other organs as multisystemic IgG4-RD, in accordance with prior studies $(7,8,10,13)$.

Immunological factors may be associated with the mechanism of IgG4-related IP. Some patients exhibited an elevated level of serum IgE (cases 1, 3 and 6), with or without allergic symptoms, and the blood inflammatory and immunological indices in these cases were raised to different degrees; for example, elevated serum CRP (cases 1-4 and 7), ESR (cases 1, 2, 4 and 7), ANA (cases 1 and 2) and IgG4 levels (cases 1-7). Lung lesions characterized by dense $\mathrm{IgG}^{+}$lymphoplasmacytic infiltration and an elevated ratio of $\mathrm{IgG}_{4} / \mathrm{IgG}^{+}$plasma cells were similar to the typical pulmonary manifestations of immune systemic diseases. In the present study, the interstitial lung disorder in these pathological specimens was initially misdiagnosed as NSIP or a tumor (cases 1-7), consistent with earlier studies $(7,10,11,20,21)$. Furthermore, the various interstitial manifestations on chest $\mathrm{CT}$ images corresponded pathologically to IP with IgG4-related fibrosis or sclerosing inflammation. Obliterative vasculitis and a high level of $\alpha-1-\mathrm{AT}$ expression were also observed in the patients enrolled in the present study. Fibrosis and vasculitis induced by trypsin-antitrypsin imbalance have been reported in immune systemic disorders, including inflammatory bowel disease (22) and hepatic fibrosis (23), and as a key initiator in IgG4-related pancreatitis (24). In addition, it has been observed that mutations in protease serine 1 cause ectopic trypsinogen activation, and calcitonin gene-associated peptide- $\beta$ splice region pathogenic variants may lead to plasma cell neurotropic enrichment, inducing obliterative vasculitis and perineural inflammation in the pancreas in type 1 AIP $(6,25)$. Vascular lesions may lead to vascular obstruction and secrete several vasoactive substances, ultimately inducing venous and fiber proliferation $(26,27)$. Finally, treatment with steroid therapy and anti-idiotypic antibodies has been confirmed to restore balance to the immune system and promote healing in IgG4-RD; however, without maintenance steroid therapy, disease relapse is common (8).

Although $\mathrm{IgG}^{+}$plasma cell infiltration and elevated serum IgG4 levels are part of the immunopathological process of $\mathrm{IgG} 4-\mathrm{RD}$, the role of $\mathrm{IgG} 4$ antibodies remains unclear. B cells differentiating into specific $\mathrm{IgG}^{+}$plasma 
cells (class-switching) were activated by particular antigens early and throughout the disease course in the immediate microenvironment (28). Specific bacterial antigens, including staphylococcal chronicity of colonization and/or (subclinical) infections, have been proposed to promote $\operatorname{IgG} 4$ responses to a larger number of pathogen-host interactions (28). During this process, the production of IgG4 antibodies appears to be driven in part by $\mathrm{T}$ helper 2 and $\mathrm{T}_{\text {reg }}$ cell cytokines, including interleukin (IL)-4, IL-5 and IL-13, as well as IL-10 and transforming growth factor- $\beta$, which may direct class-switching and ultimately promote lung fibrosis (29). IgG4 antibodies are associated with tolerance via mediating allergic responses to promote eosinophilia and IgE production (29-31). Triggering IgE-producing plasma cells is largely dependent on class-switching cytokines IL-4 and IL-5 (32). In some of the cases presented here, eosinophilic infiltration and an IP pattern at the boundaries of the nodules were observed.

The present study had certain limitations. Firstly, the immune mechanism of IgG4-related fibrosis associated with high numbers of $\operatorname{IgG}^{+}$plasma cells and high serum IgG4 level remains unknown, as most cytokines known to be associated with enhancing $\mathrm{IgG} 4$ production have similar effects on IgE production $(28,29)$. The driving force behind $\mathrm{IgG} 4$ production and the mechanism underlying the shift in balance between $\operatorname{IgG} 4$ and $\operatorname{IgE}$ also remains unclear. It is a multifactorial pathophysiological process that requires further investigation to elucidate whether other mechanisms are involved. In addition, patients with IgG4-RD are often misdiagnosed with cancer due to the tumefactive lesions and elevated levels of tumor markers. As pulmonary fibrosis is associated with an increased risk of developing lung cancer, and tumor indicators, particularly CEA, have been proven to be associated with epithelial malignancies (18), whether or not IgG4-RD confers increased susceptibility to cancer development remains to be ascertained. An additional limitation of the present study was the low number of patients recruited and the lack of complete clinical data for the majority of those patients.

In conclusion, the present study describes a series of patients to demonstrate the specific characteristics of IgG4-RILD. The results suggest that IgG4-RILD should be considered in cases of IIPs to avoid diagnostic delay and the associated increase in morbidity. In addition, the present study provides evidence that B-cell class-switching and IgG4-related immunopathological processes may be associated with the pathogenesis of pulmonary lesions. Further studies on $\mathrm{IgG} 4^{+}$plasma cell subsets are required.

\section{Acknowledgements}

The present study was supported by the National Natural Science Foundation of China (grant nos. 81571613 and 81572442), the National Key Technology R\&D Program of China (grant no. 2012AA022604), the Joint Fund for Program of Science Innovation of Fujian Province (grant no. 2016Y9011), the Natural Science Foundation of Fujian (grant no. 2015Y0015), the Nursery Foundation of Fujian Medical University (grant no. 2015MP026) and the Sail Foundation of Fujian Medical University (no. 2016QH063).

\section{References}

1. Stone JH, Zen Y and Deshpande V: IgG4-related disease. N Engl J Med 366: 539-551, 2012.

2. Peikert T, Shrestha B, Aubry MC, Colby TV, Ryu JH, Sekiguchi H, Smyrk TC, Specks U and Yi ES: Histopathologic overlap between fibrosing mediastinitis and IgG4-related disease. Int J Rheumatol 2012: 207056, 2012.

3. Umehara H, Okazaki K, Masaki Y, Kawano M, Yamamoto M, Saeki T, Matsui S, Sumida T, Mimori T, Tanaka Y, et al: A novel clinical entity, IgG4-related disease (IgG4RD): General concept and details. Mod Rheumatol 22: 1-14, 2012.

4. Yoshida K, Toki F, Takeuchi T, Watanabe S, Shiratori K and Hayashi N: Chronic pancreatitis caused by an autoimmune abnormality. Proposal of the concept of autoimmune pancreatitis. Dig Dis Sci 40: 1561-1568, 1995.

5. Kamisawa $\mathrm{T}$ and Okamoto A: Autoimmune pancreatitis: Proposal of IgG4-related sclerosing disease. J Gastroenterol 41: 613-625, 2006.

6. Gao F, Li Y, Wang C, Zhuang Z, Liu QC, Chen J, Hong G and $\mathrm{Xu} \mathrm{Z}$ : Identification of a novel frame-shift mutation in PRSS1 gene in han patients with autoimmune pancreatitis. Current Mol Med 14: 340-348, 2014.

7. Zen Y, Inoue D, Kitao A, Onodera M, Abo H, Miyayama S, Gabata T, Matsui $\mathrm{O}$ and Nakanuma Y: IgG4-related lung and pleural disease: A clinicopathologic study of 21 cases. Am J Surg Pathol 33: 1886-1893, 2009.

8. Ahn JH, Hong SI, Cho DH, Chae EJ, Song JS and Song JW: A Case of IgG4-related lung disease presenting as interstitial lung disease. Tuberc Respir Dis (Seoul) 77: 85-89, 2014.

9. Matsuo T, Ichimura K, Sato Y, Tanimoto Y, Kiura K, Kanazawa S, Okada T and Yoshino T: Immunoglobulin G4 (IgG4)-positive or -negative ocular adnexal benign lymphoid lesions in relation to systemic involvement. J Clin Exp Hematop 50: 129-142, 2010.

10. Takato H, Yasui M, Ichikawa Y, Fujimura M, Nakao S, Zen Y and Minato H: Nonspecific interstitial pneumonia with abundant IgG4-positive cells infiltration, which was thought as pulmonary involvement of IgG4-related autoimmune disease. Intern Med 47: 291-294, 2008.

11. Inoue D, Zen Y, Abo H, Gabata T, Demachi H, Kobayashi T, Yoshikawa J, Miyayama S, Yasui M, Nakanuma Y and Matsui O: Immunoglobulin G4-related lung disease: CT findings with pathologic correlations. Radiology 251: 260-270, 2009.

12. Tanaka K, Nagata K, Tomii K and Imai Y: A case of isolated IgG4-related interstitial pneumonia: A new consideration for the cause of idiopathic nonspecific interstitial pneumonia. Chest 142: 228-230, 2012.

13. Taniguchi T, Ko M, Seko S, Nishida O, Inoue F, Kobayashi H, Saiga T, Okamoto M and Fukuse T: Interstitial pneumonia associated with autoimmune pancreatitis. Gut 53: 770-771, 2004.

14. Umehara H, Okazaki K, Masaki Y, Kawano M, Yamamoto M, Saeki T, Matsui S, Yoshino T, Nakamura S, Kawa S, et al: Comprehensive diagnostic criteria for IgG4-related disease (IgG4-RD) 2011. Mod Rheumatol 22: 21-30, 2012.

15. Yamamoto M, Takahashi H, Suzuki C, Tabeya T, Ohara M, Naishiro Y, Yamamoto H, Imai K and Shinomura Y: Analysis of serum IgG subclasses in Churg-Strauss syndrome-the meaning of elevated serum levels of IgG4. Intern Med 49: 1365-1370, 2010.

16. Sato Y, Notohara K, Kojima M, Takata K, Masaki Y and Yoshino T: IgG4-related disease: Historical overview and pathology of hematological disorders. Pathol Int 60: 247-258, 2010.

17. Mikolasch TA, Garthwaite HS and Porter JC: Update in diagnosis and management of interstitial lung disease. Clin Med (Lond) 16 (Suppl 6): S71-S78, 2016.

18. Fahim A, Crooks MG, Wilmot R, Campbell AP, Morice AH and Hart SP: Serum carcinoembryonic antigen correlates with severity of idiopathic pulmonary fibrosis. Respirology 17 : 1247-1252, 2012.

19. Travis WD, Costabel U, Hansell DM, King TE Jr, Lynch DA, Nicholson AG, Ryerson CJ, Ryu JH, Selman M, Wells AU, et al: An official American thoracic society/european respiratory society statement: Update of the international multidisciplinary classification of the idiopathic interstitial pneumonias. Am J Respir Crit Care Med 188: 733-748, 2013. 
20. Shrestha B, Sekiguchi H, Colby TV, Graziano P, Aubry MC, Smyrk TC, Feldman AL, Cornell LD, Ryu JH, Chari ST, et al: Distinctive pulmonary histopathology with increased IgG4-positive plasma cells in patients with autoimmune pancreatitis: Report of 6 and 12 cases with similar histopathology. Am J Surg Pathol 33: 1450-1462, 2009.

21. Ryu JH, Sekiguchi H and Yi ES: Pulmonary manifestations of immunoglobulin G4-related sclerosing disease. Eur Respir J 39: 180-186, 2012.

22. Papanikolaou I, Kagouridis K and Papiris SA: Patterns of airway involvement in inflammatory bowel diseases. World J Gastrointest Pathophysiol 5: 560-569, 2014.

23. Hidvegi T, Ewing M, Hale P, Dippold C, Beckett C, Kemp C, Maurice N, Mukherjee A, Goldbach C, Watkins S, et al: An autophagy-enhancing drug promotes degradation of mutant alpha1-antitrypsin $\mathrm{Z}$ and reduces hepatic fibrosis. Science 329: 229-232, 2010.

24. Lin K, Gao F, Chen Q, Liu Q and Chen S: Framework for Interpretation of Trypsin-antitrypsin Imbalance and Genetic Heterogeneity in Pancreatitis. Saudi J Gastroenterol 21: 198-207, 2015.

25. Liu QC, Chen F, Wu CY, Gao F, Zhuang ZH, Chen JT, Cai B, Zhang T, Guo L, Lin LQ, et al: CALCB splice region pathogenic variants leading to plasma cell neurotropic enrichment in type 1 autoimmune pancreatitis. Cell Death Dis 8: 2591, 2017.

26. Giacomelli R, Liakouli V, Berardicurti O, Ruscitti P, Di Benedetto P, Carubbi F, Guggino G, Di Bartolomeo S, Ciccia F, Triolo G and Cipriani P: Interstitial lung disease in systemic sclerosis: Current and future treatment. Rheumatol Int 37: 853-863, 2017
27. Okamoto A, Nojiri T, Konishi K, Tokudome T, Miura K, Hosoda H, Hino J, Miyazato M, Kyomoto Y, Asai K, et al: Atrial natriuretic peptide protects against bleomycin-induced pulmonary fibrosis via vascular endothelial cells in mice: ANP for pulmonary fibrosis. Respir Res 18: 1, 2017.

28. Swierstra J, Debets S, de Vogel C, Lemmens-den Toom N, Verkaik N, Ramdani-Bouguessa N, Jonkman MF, van Dijl JM, Fahal A, van Belkum A and van Wamel W: IgG4 subclass-specific responses to Staphylococcus aureus antigens shed new light on host-pathogen interaction. Infect Immun 83: 492-501, 2015.

29. Nirula A, Glaser SM, Kalled SL and Taylor FR: What is IgG4? A review of the biology of a unique immunoglobulin subtype. Curr Opin Rheumatol 23: 119-124, 2011.

30. Maguire GA, Kumararatne DS and Joyce HJ: Are there any clinical indications for measuring IgG subclasses? Ann Clin Biochem 39: 374-377, 2002.

31. Aalberse RC, van der Gaag R and van Leeuwen J: Serologic aspects of IgG4 antibodies. I. Prolonged immunization results in an IgG4-restricted response. J Immunol 130: 722-726, 1983.

32. Hussain R, Poindexter RW and Ottesen EA: Control of allergic reactivity in human filariasis. Predominant localization of blocking antibody to the IgG4 subclass. J Immunol 148: 2731-2737, 1992.

(i) $(9)$ This work is licensed under a Creative Commons Attribution-NonCommercial-NoDerivatives 4.0 International (CC BY-NC-ND 4.0) License. 